\title{
RESET
}

Recherches en sciences sociales sur Internet

9| 2020

Les pratiques religieuses et internet

\section{Former une ambiance sacrée}

L'élaboration de « cahiers de prières » et les pratiques dévotionnelles de jeunes brésiliennes sur YouTube

Staging a sacred ambiance. The production of prayer books and devotion

practices of young Brazilian women on YouTube

\section{Kelber Pereira Gonçalves}

\section{(2) OpenEdition}

Journals

Édition électronique

URL : https://journals.openedition.org/reset/2287

DOI : $10.4000 /$ reset.2287

ISSN : 2264-6221

Éditeur

Association Recherches en sciences sociales sur Internet

Référence électronique

Kelber Pereira Gonçalves, « Former une ambiance sacrée », RESET [En ligne], 9 | 2020, mis en ligne le 06 octobre 2020, consulté le 20 mai 2021. URL : http://journals.openedition.org/reset/2287 ; DOI : https://doi.org/10.4000/reset.2287

Ce document a été généré automatiquement le 20 mai 2021.

(c) Association Recherches en sciences sociales sur Internet 


\title{
Former une ambiance sacrée
}

L'élaboration de «cahiers de prières » et les pratiques dévotionnelles de jeunes brésiliennes sur YouTube

\author{
Staging a sacred ambiance. The production of prayer books and devotion \\ practices of young Brazilian women on YouTube
}

Kelber Pereira Gonçalves

1 L'internet a transformé non seulement les logiques des institutions religieuses mais aussi la manière dont la religion et les pratiques religieuses peuvent être pensées dans un contexte de "modernité religieuse" (Hervieu-Léger, 1997). Si de nombreux chercheurs du champ d'études en communication et religion (et plus précisément en communication religieuse et numérique) se sont intéressés aux institutions et à l'usage de l'internet comme médium de transmission d'un message religieux, le nombre de travaux sur les pratiques religieuses populaires et syncrétiques reste encore minoritaire (Campbell \& Vitullo, 2016). Cette recherche s'intéresse précisément au processus de mise en scène d'une "ambiance sacrée " à travers des productions audiovisuelles publiées et diffusées par de jeunes femmes laïques chrétiennes brésiliennes sur la plateforme YouTube. Ces productions sous forme de «tutoriels » ont pour ambition d'apprendre aux internautes à élaborer un cahier de prières ou un journal spirituel et de les inciter, par ce biais, à adopter une pratique dévotionnelle chrétienne quotidienne. Cette pratique spirituelle - dont l'usage de cahiers de prières ${ }^{1}$ est le point-clef - correspond, d'après les internautes, au temps quotidien qu'elles consacrent à prier et qui se trouve donc réservé afin d'être en «communion avec Dieu ».

2 L'intérêt que nous portons aux cahiers de prières trouve son origine dans une recherche portant sur les usages du numérique en contexte religieux qui mobilisait l'observation ethnographique non participante. Elle a été réalisée entre les années 2016 et 2018 auprès d'une communauté charismatique catholique au Brésil. Lors de nos observations, nous avons relevé l'usage des cahiers de prières par certains des fidèles pendant les séances de prières. Bien que les cahiers de prières n'étaient pas l'objet de cette recherche, nous nous sommes intéressés à cette pratique dont nous ignorions 
jusque-là l'existence. Nous avons alors effectué quelques recherches en ligne à son propos et nous avons vite repéré une multitude de références. En saisissant le terme cadernos de oração (cahier de prières) sur le moteur de recherche de la version brésilienne de Google, au moment même de l'élaboration de ce présent texte, nous pouvons trouver environ 4390000 résultats entre textes, images et contenus audiovisuels. Bien que le terme soit largement présent dans les moteurs de recherche, l'intérêt public pour les cadernos de oração ne trouve pas d'écho, actuellement, dans la recherche en sciences humaines et sociales, cette pratique de piété demeurant jusqu'à présent très peu étudiée. Compte tenu de leur caractère pédagogique, les contenus audiovisuels publiés sur la plateforme YouTube sous le format tutoriel ont été choisis pour composer notre corpus. Ainsi, nous avons saisi les mots clefs caderno de oração (cahier de prières) sur le moteur de recherche de la plateforme Youtube et cliqué sur la première vidéo proposée automatiquement par le site à partir de l'option "lecture automatique». À la suite de la visualisation de sept productions audiovisuelles, élaborées par sept youtubeuses, nous avons pu établir suffisamment d'éléments décor, rythme, acteurs, présence ou absence de son, scénario etc. - nous permettant d'élaborer notre analyse. Bien que les dynamiques liées aux questions algorithmiques concernant la suggestion automatique des vidéos par la plateforme mériteraient d'être aussi étudiées (Cardon, 2015), elles dépassent largement le cadre de ce travail. C'est pourquoi ces dynamiques ne sont ici pas prises en compte. Nous allons, pour notre analyse, tout d'abord, décomposer les vidéos et les décrire et ensuite, établir et comprendre les relations entre les éléments décomposés, c'est-à-dire, interpréter (Vanoye \& Goliot-Lété, 2006) afin de tenter de saisir comment une ambiance devient " sacrée » et se trouve mise en scène dans ces vidéos.

3 Pour notre analyse, nous avons eu recours à un cadre théorique qui conjugue principalement les usages des technologies de l'information et de la communication en contexte religieux (Douyère, 2011, 2014, 2018 ; Campbell, 2005, 2010 ; Helland, 2005), et l'anthropologie des espaces (Thibaud, 2012, 2018; Bégout, 2018). L'intérêt porté aux pratiques religieuses non institutionnalisées sur internet se justifie, à notre sens, d'une part, par le phénomène de crise du concept de religion (Steil \& Toniol, 2013) et de l'effacement des autorités religieuses légitimées par les institutions et, d'autre part, en raison de la croissance ou de l'explosion de la subjectivité religieuse (Hervieu-Léger, 2010). Ces dynamiques font, depuis quelques années déjà, de l'espace numérique un " endroit » riche pour l'étude de pratiques religieuses profondément hétérogènes.

Nous proposons une présentation de cette recherche en quatre temps. Dans un premier temps, nous tenterons de mettre en relation la prière et les ambiances qui y sont consacrées, et nous procèderons à un très bref rappel historique de l'usage des cahiers de prières, des journaux intimes ou des guides spirituels dans ce que nous pourrions qualifier de tradition chrétienne ancienne. Dans un deuxième temps, nous allons nous intéresser à la définition et à l'usage des cahiers de prières, en partant de la conception des youtubeuses étudiées dans notre corpus. Dans un troisième temps, nous chercherons à mieux comprendre les méthodologies mises en place pour l'élaboration des cahiers de prières, notamment en ce qui concerne l'organisation des cahiers et leur esthétique. Enfin, dans un quatrième temps, nous mettrons en exergue les stratégies mises en place dans la constitution d'une ambiance sacrée ou de l'espace figuratif du sacré dans les productions audiovisuelles étudiées. 


\section{Des cahiers et des prières}

5 La prière est un terme polysémique : elle recouvre une variété de formes, de styles, de tournures et de "manières de faire». La prière peut être accompagnée d'interrogations, de demandes, de remerciements. Elle peut faire partie intégrante de la pénitence, voie et outil par laquelle le croyant peut être délivré du pêché préalablement commis. Elle peut être dite à voix haute, déclamée, chantée, écrite, accompagnée d'images ou de sons. Elle peut se faire en groupe, ou dans une communauté, parfois dans des espaces qui lui sont réservés; elle peut être récitée, répétée, dite et redite (sous forme de litanie), assortie d'objets conçus à cette fin ou qui lui sont associés (un chapelet, un prie-Dieu, des iconographies, un scapulaire, un cierge, une image de piété ou autre). La prière, ce moment de communication entre le croyant et le dieu exige le plus souvent un espace-temps qui lui soit propre : un lieu de culte, dans l'espace intime de sa maison, durant une période de neuf jours (une neuvaine), ou encore lors d'une période plus longue de recueillement et de réflexion consacrée à la prière et lors du travail spirituel $\mathrm{du}$ carême. Elle peut se faire de manière plus confidentielle, individuellement, dans la solitude et le silence de la clôture, mais aussi par l'introspection durant le temps de transport, lorsque le croyant - entourée d'inconnus - se rend au travail, ou en revient. L'on prie le dieu chrétien pour soi et pour les autres. On prie pour obtenir des "grâces » : pour la guérison d'une maladie, pour trouver un emploi, pour trouver l'âme sœur, pour réussir un examen ou bien pour remercier des grâces déjà obtenues. Pour ce qui est de l'Église catholique, l'on peut prier également les saints - ou «avec » eux - (dulie), ou demander l'intercession de la vierge Marie auprès de Dieu. L'on peut prier à l'intention des vivants, mais aussi pour les morts, afin que leur âme soit en paix - pour celles qui sont plus proches de Dieu, mais aussi (voire principalement) pour celles qui se trouveraient, en contexte chrétien, au purgatoire ou en enfer.

6 Exégète et philosophe prolifique des textes sacrés, Origène considérait que l'Écriture avait trois sens (littéral, moral et spirituel) qui correspondraient aux trois parties de l'homme, à savoir, le corps, l'âme et l'esprit. Origène comprenait donc l'écriture et la prière comme deux entités indissociables. Dans De la prière, différentes manières de prier ont été théorisées par le religieux qui considérait que le fidèle devrait « inscrire trois fois dans sa propre âme les pensées des saintes Écritures » (Origène, $2012: 172)$. En autre, l'exégète a élaboré encore les principes de la lectio divina (la lecture sainte), méthode de lecture des saintes Écritures et de prière répandue formellement ensuite en Occident par Ambroise de Milan. La Lectio divina, conjointement à l'obéissance, à l'humilité, au travail et à la liturgie monastique, a constitué, des siècles plus tard, les ingrédients de la vie ascétique dans les monastères du Moyen Âge en Europe. Cette méthode pieuse a été particulièrement utilisée et diffusée en contexte français par Guingues II le Chartreux, prieur du monastère de La Grande Chartreuse (Trottmann, 2003). D'autres ouvrages importants traitant des pratiques de piété liées à l'écriture s'inscrivent dans une tradition chrétienne aussi ancienne. Les Confessions, largement étudiées, peuvent être appréhendées à la fois comme étant un journal intime ou un journal spirituel et un manuel pédagogique pour dire la prière. Écrit par Augustin d'Hippone, ce texte n'est pas seulement la confession de ses péchés, mais aussi un témoignage au service d'une " pédagogie de transmission de la foi » (Pierron, 1995 : 265). Aussi, des méthodologies de la prière, ayant recours à tout le corps et aux sens, figurent 
également dans le manuscrit Neuf manières de prier de saint Dominique. Ces formes de prières, censées avoir été pratiquées et élaborées par Dominique de Guzman, constituent «une représentation incitative et identitaire de la prière et du recueillement " (Douyère, 2014) et s'inscrivent également comme un guide pour une méthodologie de la pratique pieuse de la prière. Il s'agit d'un mode de prière destiné à être imité. En ce sens, ce manuscrit est considéré par les dominicains, non seulement comme un document hagiographique, mais aussi un outil pédagogique sous forme de Manuale Precum (Palazzo, 2016, 2017). Au cours de la Renaissance, les Exercices Spirituels élaborés par Ignace de Loyola, fondateur de la Compagnie de Jésus, illustre une fois de plus une tradition chrétienne à élaborer des guides pédagogiques de prière. Loyola donne une définition de la notion de "discernement »: le discernement ne pourrait être acquis qu'à partir des lectures des Saintes Écritures et de la pratique des exercices spirituels. Grâce à la "pédagogie de l'intelligence ", le discernement se construit à partir d'un "vase incessamment communicant entre vie intellectuelle et expérience spirituelle " (Sevez, 2018 : 79). Cette notion élaborée par Ignace de Loyola représente donc une nouveauté et agit comme une idée maîtresse ; elle demeure d'actualité dans la chrétienté de tradition catholique.

\section{Le cahier de prières, pilier de la pratique dévotionnelle}

7 Pour ce qui est du rapport entre écriture et prière en contexte chrétien brésilien, nos recherches n'ont pas permis d'établir avec certitude l'origine de l'usage des cahiers de prières comme outil "d'organisation" de la prière et comme objet ou artefact de support à cette pratique de piété. Si nous ne pouvons pas affirmer que le manque d'intérêt scientifique pour les cahiers de prières découle, du moins en partie, de son caractère populaire, nous pouvons soutenir que les cahiers de prières étaient utilisés dans ce pays au moins dès les premières décennies du siècle dernier. Une recherche réalisée par Santana en 2011, intitulée Entre Bordados, Cadernos e Orações (entre broderie, cahiers et prières) portant sur les pratiques éducatives, pédagogiques et didactiques au sein d'un orphelinat catholique pour jeunes filles dans la ville de São Cristovão, au Brésil, a montré que l'élaboration et l'usage des cahiers de prières était une pratique courante: cette pratique faisait notamment corps avec le fonctionnement global de l'institution entre les années 1922 et 1969. En effet, la recherche fait apparaître, à partir des récits des ex-professeures et étudiantes, que l'usage des cahiers de prières - et l'élaboration d'une " poésie religieuse » qui pouvait s'y inscrire - était une pratique suscitée par la direction de l'Institution. Comme l'indique le titre de la recherche, conjointement à l'emploi des cahiers de prières, des cours de broderie, des cours de "bonnes manières" simultanément à l'enseignement religieux (programmes d'oraisons et participation quotidienne à la messe) constituaient les ingrédients nécessaires pour assurer une "bonne " formation pour les femmes selon les perceptions de l'époque dans le nord-est brésilien. Dans ce contexte, le modèle institutionnel était ancré davantage dans le fait de «l'éduquer » plutôt que dans celui d'« instruire", " afin de leur donner les conditions nécessaires pour remplir un rôle social satisfaisant, c'est-à-dire, d'être des bonnes mères et des femmes au foyer exemplaires » (Santana, $2011: 23$ ).

8 L'usage de l'écriture pour assister la prière, l'emploi des exercices spirituels ainsi que l'usage de cahiers comme manuels de piété et comme outils pour favoriser une 
communication - comprise ici comme "la production et mise en circulation de sens " (Douyère, 2018 : 19) - avec Dieu, accompagne l'histoire de la chrétienté dès les premiers siècles. L'usage de cahiers de prières a non seulement traversé le temps, mais retrouve un certain succès, du moins si l'on se reporte aux nombreuses références présentées par le moteur de recherches Google ainsi que par la plateforme YouTube. D'après les youtubeuses étudiées dans notre corpus, l'usage du cahier de prières s'inscrit dans une logique de communion avec Dieu.

Dans notre étude, nous n'avons pas eu accès aux données relatives au profil sociologique de ces femmes sur leur chaîne YouTube ou à partir des productions visionnées. Pour ce qui est de leur âge, nous avons choisi d'utiliser le terme «jeunes femmes » car nous supposons qu'il s'agit d'internautes ayant entre 15 et 35 ans environ. En ce qui concerne la filiation religieuse des youtubeuses étudiées, à l'exception de Sarah Néry, qui précise faire partie de la communauté charismatique catholique Shallon $^{2}$, les autres jeunes femmes ne déclarent pas dans les vidéos analysées leur appartenance religieuse. Par leur vocabulaire, nous supposons qu'il s'agit de femmes évangéliques ${ }^{3}$ (elles parlent de culte et non de messe par exemple). D'ailleurs, dans les commentaires laissés par d'autres internautes, nous pouvons constater des messages d'autres jeunes filles se déclarant catholiques et aussi évangéliques tenant à féliciter la youtubeuse pour sa vidéo. Cela confirme une tendance de rapprochement entre les catholiques (principalement représenté par le renouveau catholique) et évangéliques ces dernières années au Brésil - mais aussi dans d'autres parties du globe. Par ailleurs, cette alliance sous la forme d'un "œcuménisme chrétien" lorsque des intérêts communs sont en jeu, est visible notamment dans des pratiques religieuses, mais aussi dans la politique ou dans l'offre et la consommation de produits culturels religieux.

Selon Francine Veríssimo, dans sa vidéo intitulée 5 motivos para começar um diário espiritual e (3 dicas de como fazê-lo! $)^{4}$, le cahier de prières peut être défini comme un «journal intime» ou «journal spirituel», entendu comme un outil pédagogique d'affermissement de la foi et un médium qui serait à même de rapprocher les croyants de Dieu : «Premièrement, le journal spirituel t'aide à organiser et donner de la clarté à tes pensées. L'écriture a ce pouvoir de nous aider à catégoriser nos pensées $\left(00^{\prime} 22^{\prime \prime}-00^{\prime} 48^{\prime \prime}\right)$. Deuxièmement, le journal spirituel t'aide à apprendre davantage sur la parole de Dieu. Par-dessus tout, le journal spirituel est un outil d'apprentissage " (00'53"-00' 57"). Il s'agit, d'un outil de communication pour « discuter avec Dieu », ditelle. Elle souligne encore que c'est à travers et grâce au cahier de prières qu'il est possible «d'écrire les versets qui nous ont sauté aux yeux un tel jour, ce que nous sommes en train d'apprendre à l'Église, les messages ou ce que le Seigneur, à travers le Saint-Esprit nous parle à notre cœur. Il est une espèce de cahier dans notre école de la grâce. C'est avec lui que nous allons grandir en parfaite connaissance de notre Seigneur et sauveur Jésus Christ» $\left(00^{\prime} 50^{\prime \prime}-01^{\prime} 23 "\right)$. Le cahier de prières, toujours d'après Francine Veríssimo, "t'aide autant à parler qu'écouter le Christ, puisqu'après tout, tu vas écrire ce qu'il va te dire par sa parole, ce qu'il sera en train de chuchoter dans ton cœur, et toi, tu vas aussi écrire tes prières, tes sentiments » (1'36"-1'48"). La youtubeuse Tainá Brito dans la vidéo intitulée Como eu faço meu devocional / \#semi $i^{5}$ explique que " quand on écrit [ça] devient encore plus réel. C'est comme si c'était un document [qui indique ce que] je dois changer dans un domaine car Dieu m'en a parlé aujourd'hui » ( $\left.3^{\prime} 40^{\prime \prime}-3^{\prime}: 48^{\prime \prime}\right)$. Ainsi, pour ces jeunes filles chrétiennes, la prière est comprise comme modus operandi de communication avec une entité invisible supposée communiquer, par «l'Écriture », ainsi appropriée; ce n'est nullement une communication 
intrapersonnelle. Le croyant ne parle pas à soi-même, mais il parle à «dieu ». Ainsi il s'agirait non pas d'un monologue, mais d'un dialogue et donc d'un acte de communication entre la personne du croyant et le dieu qui communique avec le croyant.

11 L'usage du cahier de prières constitue la pièce fondamentale dans la pratique dévotionnelle quotidienne cultivée par ces jeunes femmes. Dans la vidéo intitulée $O$ que é? E como fazer: Devocional ${ }^{6}$, Michelle Souza nous explique que cette pratique dévotionnelle consiste à « réserver une période significative de la journée pour être en communion avec Dieu »; [Il s'agit de] « prendre du temps pour discuter avec lui, pour lire sa parole, l'étudier, c'est-à-dire, réserver du temps pour adorer Dieu » (00'32"-00'49"). Autrement dit, il s'agit de prendre « au moins une petite heure de la journée pour la consacrer à Dieu, pour garder du temps, pour étudier ses écritures, pour garder du temps de discuter [...] avec lui » (0'50"-1'21"). Michelle de Souza met encore en exergue ce qu'elle entend par le terme "significatif» dans la pratique dévotionnelle quotidienne : « un temps significatif qui ne consisterait pas à 'dire Dieu merci pour la journée, amen' à la fin de la journée ou au moment de se coucher » $\left(0^{\prime} 55^{\prime \prime}-0^{\prime} 59^{\prime \prime}\right)$. Bien qu'il n'y ait pas de manière unique de se livrer à cette pratique dévotionnelle ou d'élaborer des cahiers de prières, un certain nombre d'éléments doivent être réunis pour favoriser ce « dialogue » avec Dieu.

La vidéaste Tainá Brito explique pour sa part que «la dévotion est quelque chose de très important dans nos vies » (7'49"-7'52"). Michelle Souza souligne de son côté qu'il est tout d'abord question de « commencer par demander au Saint-Esprit de nous guider dans les Écritures » (4'33"-4'36"). Puis, il est question de « choisir un horaire et faire de cet horaire une habitude, ainsi qu'un endroit tranquille, un petit coin qui ne soit qu'à toi » (2':20"-2'23"). Michelle explique encore qu'il est important de consacrer un moment pour la dévotion, en dépit du manque de temps quotidien. Pour les personnes qui travaillent, elle souligne, à titre d'exemple, que la pause du déjeuner peut constituer un moment propice pour la réalisation de la pratique dévotionnelle quotidienne ainsi que pour l'exercice de l'écriture de la prière. Michelle explique encore préférer réserver la première heure de sa journée : «lorsque je me réveille je cherche à faire ma dévotion, parce que j'aime m'élever et commencer de suite à discuter avec Dieu» (1'49"-1'56"). Tainá Brito explique que la dévotion peut être réalisée à partir d'une méthode représentée par l'acronyme anglais «SOAP » (Script, Observation, Application, Prayer). À la suite de la lecture d'un passage, d'un chapitre ou d'un verset biblique, les fragments considérés par le croyant les plus importants peuvent être réécrits (script); ensuite, le fidèle peut résumer avec ses propres mots ce que, d'après elle, «Dieu a voulu dire ", dans cette étape de l'observation (Observation); la troisième étape consisterait à réfléchir à comment employer de manière pratique la " vérité » de la parole de Dieu dans la vie quotidienne du croyant (Application) ; enfin, la pratique dévotionnelle peut être clôturée par la prière proprement dite et s'inspirer du contenu lu par le croyant dans les Saintes Écritures (prayer).

\section{La chambre : les méthodologies de prière dans l'espace figuratif du sacré}

Dans le cas des youtubeuses, les productions audiovisuelles du genre "tutoriel " s'inscrivent d'emblée dans une démarche qui vise à transmettre ou à «faire savoir » à l'autre quelque chose d'un fait socialement construit au préalable et compris comme 
une réalité par ces jeunes filles, à savoir, «l'action» de Dieu dans leurs vies. Cette démarche de transmission de connaissances suppose aussitôt le recours à des méthodes mises en place en ayant pour but d'accomplir une ou un ensemble de tâches. La transmission de savoirs et le caractère pédagogique que revêtent ces vidéos sous forme de tutoriel sont manifestes par l'intitulé même de ces productions audio-visuelles: "Comment je fais ma dévotion", "Comment j'organise mon cahier de prières", "Comment je fais ma dévotion en pratique», "Comment j'utilise mon cahier de dévotion » ou encore « Cinq raisons pour commencer un journal spirituel ».

Pour ce qui est de la méthodologie de la lecture de la Bible, et par conséquent, la lecture des contenus qui serviront de fondement aux contenus du cahier de prières même, Tainá Brito explique que sa Bible est "un petit peu colorée » (04'50"-04'58"). Cette méthodologie d'organisation de la lecture des Écritures sacrées à partir des couleurs variées permettrait donc de se repérer plus facilement dans les versets ou chapitres de la Bible. L'usage de crayons de couleurs se fait, d'après la youtubeuse, parce que «le stabilo est trop fort et la page de la Bible est toute fine, donc les crayons de couleur ne tâchent pas l'autre côté, [à savoir, le verso] ça c'est le meilleur côté !» (05'10"-05'25"). La méthodologie pour une "économie de la prière " mise en place pour faciliter la communion avec Dieu n'annule pas l'aspect esthétique dans la confection des cahiers de prières : «la commande religieuse, le don pieux, la prière ne sont pas incompatibles avec la recherche de la qualité artistique et avec le plaisir esthétique » (Christin, 2002 : 90). Toute l'histoire de la chrétienté en témoigne : on y retrouve à maintes occasions de recourir au « beau » et à l'esthétique dans les manières de prier le dieu chrétien.

À titre d'exemple, dans sa recherche anthropologique sur la prière chrétienne, Palazzo montre que l'acte de peindre au Moyen Age pouvait être équivalent à l'acte même de prier. Celui qui peignait pouvait être perçu en tant qu'homo agens qui, par sa capacité créatrice et par ses habiletés artistiques, imitait en quelque sorte le créateur. L'idée d'artiflex complète donc la « dimension artisanale de l'acte de prière utilisant des outils spécifiques tel qu'un artisan a besoin des outils adaptés dans la réalisation de son travail » (Palazzo, 2016:56). En contexte chrétien, cette mise en relation « d'intimité » ou de liaison entre le croyant et le dieu à travers la prière est particulièrement favorisée par la forme humaine du dieu chrétien. À la fois humain et divin, la figure de Jésus-Christ sous forme de verbe incarné serait susceptible d'une part de " comprendre » les misères et limitations de la matière ou de la chair et de « percevoir et être perçu » (Gauchet, 1981 cité dans Salatko, $2017: 167$ ) et, d'autre part (et par conséquent), rendre plus accessible la communication avec le Père. Le Saint-Esprit, quant à lui, serait la pièce qui complète le schéma communicationnel : il n'est, dans la conception des chrétiens, pas seulement source d'inspiration, mais agent et médium par lequel le dieu (sous la forme du père ou du fils) se communiquerait aux croyants. 
Figure : La youtubeuse Duda Ruiz dans la vidéo intitulée « Dévotionnelle avec art 6 - mon cahier de dévotionnelles » (consulté le 12/08/19).

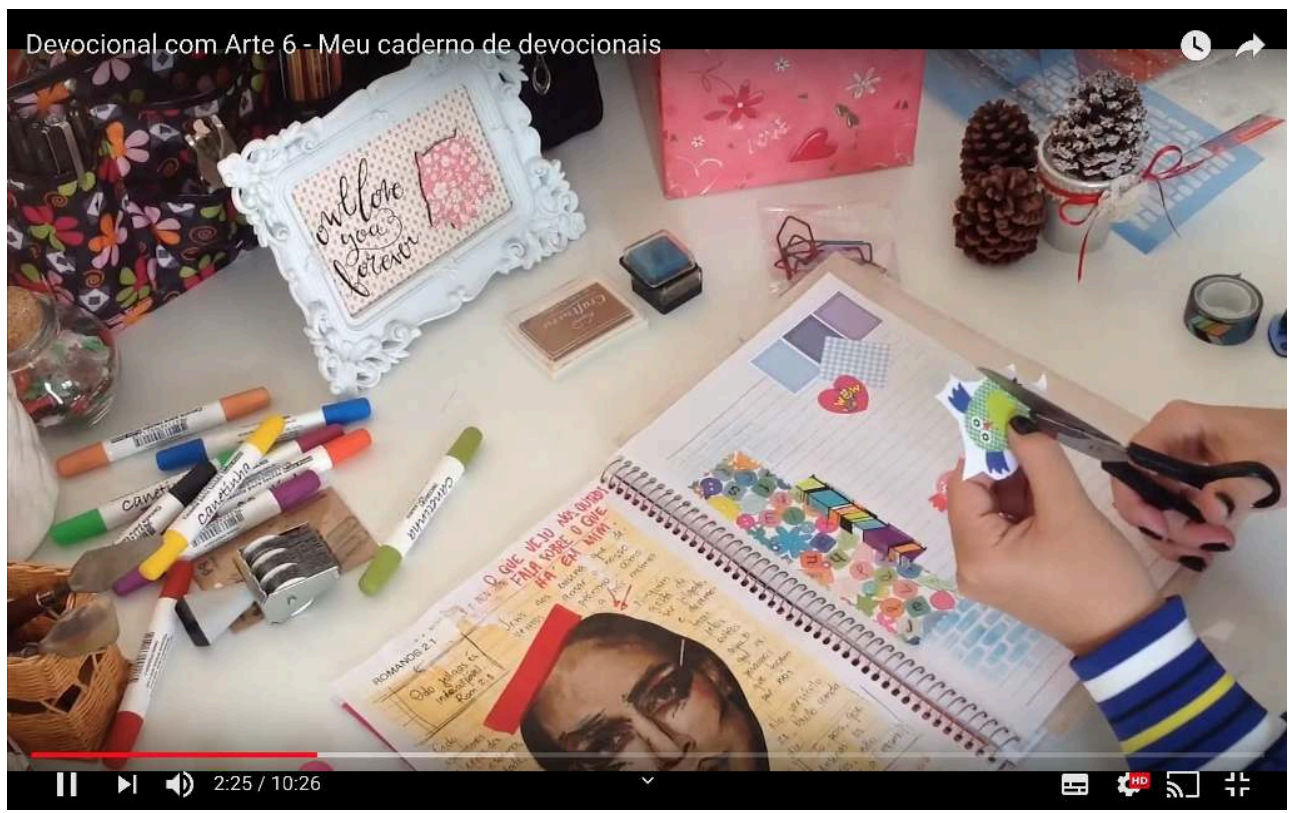

De la même manière, il n'y aurait pas qu'une seule méthode à suivre dans l'élaboration $\mathrm{du}$ cahier de prières. Ce point rejoint une fois de plus les questions évoquées précédemment à propos de l'individualisation des croyances, des conceptions religieuses et des pratiques hétérogènes, qui sont autant de caractéristiques de la modernité religieuse. Sarah Néry dans la vidéo intitulée Como eu organizo meu caderno de oração / sete categorias ${ }^{7}$, révèle que son cahier de prières est arrivé à sa fin et que ce serait donc la bonne occasion d'expliquer comment elle organise son cahier de prières à partir d'un nouveau cahier vierge. Sarah Néry explique que les pages du cahier de prières sont datées, et les récits sont accompagnés des initiales EB ou OP : EP pour étude biblique, en référence aux textes des écritures sacrées; OP pour oraison personnelle. Pour ce qui est des catégories du cahier de prières, la youtubeuse indique que son cahier de prières compte sept divisions : par exemple, la première partie est intitulée cahier de prières, groupe de prières, confession, «accomp's " $^{8}$, dates importantes, surprises de Dieu et discernement - notion visiblement inspirée de la spiritualité ignacienne. Fabíola Keffer, pour sa part, souligne dans la vidéo intitulée Devocional diário - como eu faço meu devocional na pratica ${ }^{9}$ que durant ses lectures bibliques, elle met en place une forme de méditation qui consiste à « s'imaginer dans l'histoire » ce qui renvoie une fois de plus à des techniques de prières très anciennes élaborées par des pères de l'Église ou des théologiens et qui est, notamment, assez proche de la notion de « vision imaginaire » élaborée par Ignace de Loyola (De Koninck, 2007). Fabíola Keffer ne mentionne pas le terme « cahier de prières ", mais parle plutôt de " cahier de dévotion ». Elle possède deux cahiers : un cahier de dévotion et un cahier d'études bibliques. Le cahier de dévotion se fait dans un agenda. À ce propos, elle souligne : « ce qui est bien dans le fait de le faire dans l'agenda, c'est que tu peux suivre les jours que tu l'as fait [la dévotion] dans l'agenda, les jours où tu ne l'as pas fait ». (2'41"-2'50"). L'usage de l'agenda, comme cahier de prières même, illustre, à notre sens, un « engagement». Cet ordonnancement et agencement de la pratique dévotionnelle reflètent l'existence d'un "compromis » quotidien entre le croyant et Dieu. Cela est rendu possible, d'après la youtubeuse, par la forme de l'agenda - présentant les jours 
de la semaine, les mois et l'année et des horaires - qui est censée favoriser une « organisation" des tâches de la vie quotidienne, dont la pratique dévotionnelle fait partie.

Des techniques d'artisanat peuvent être utilisées dans la perspective de "rendre beau " le cahier de prières. L'aspect pédagogique, donc relevant des méthodes et des pratiques à adopter pour y parvenir, se retrouve aussi dans l'indication des matériaux utilisés par les youtubeuses, lorsqu'elles ont recours à des techniques d'artisanat dans la confection des cahiers de prière. Duda Ruiz fait connaître aux internautes, tout à la fin de sa vidéo intitulée Devocional com Arte 5. Como eu uso o meu caderno de devocionais - Devotional Journal ${ }^{10}$ les matériels et outils de dessin utilisés pour l'ornement et le coloriage de son cahier de prières, à savoir, aquarelle et pinceau de la marque allemande Faber-Castell, papier de découpage, adhésifs, étiquettes et stylo Uni Pin 0.01 résistant à l'eau. Duda Ruiz explique encore dans la suite de sa vidéo précédente, Devocional com Arte 6 - Meu caderno de devocionais ${ }^{11}$, que d'autres matériaux peuvent être également utilisés tels que des adhésifs, des trombones, des ficelles ou des marque-pages, rapprochant l'élaboration du cahier de prière du scrapbooking. L'esthétique de la couverture du cahier de prières ainsi que sa forme ou sa taille semblent, du point de vue des youtubeuses, être également être pris en compte. Sarah Néry, pour sa part, possède deux cahiers de prières. Le premier comprend des contenus qui relèveraient essentiellement des pratiques religieuses s'inscrivant dans des vécus directement liés à la communauté religieuse dont elle fait partie. Le second est destiné à transcrire le vécu de sa pratique dévotionnelle quotidienne. Ce dernier cahier est, d'après elle, «plus petit, plus mignon et bien plus spiritualisé et bien plus oracional, (orant)» dit-elle (elle baisse alors la tête, ferme les yeux et suggère un geste, les mains jointes, traditionnellement signe d'intériorisation de la foi, en évocation de la prière ou d'humilité devant Dieu). La prière, tout comme la religion, est tout d'abord un phénomène social, non seulement par son contenu, mais aussi par sa forme : «Elle n'existe pas en dehors d'un rituel» (Mauss, 1968: 19). Bien qu'elle ne développe pas davantage ce qu'elle entend par " plus orant ", il semble visiblement que la couverture et la forme influent sur la conception que se fait la youtubeuse de la «sacralité » des cahiers. Ces pratiques combinent donc, le plus souvent, des valeurs et des normes institutionnalisées, que nous appelons doctrines ou traditions, à des perceptions très individualisées de croyances religieuses construites de manière empirique par les individus, liées à une esthétique (accompagnant la réalisation de la pratique orante) et qui sont associées à des contextes historiques, des espaces géographiques et des contextes culturels qui leur sont spécifiques. 
Figure 2 : La youtubeuse Sarah Néry dans la vidéo intitulée " comment j'organise mon cahier de prière - Sept catégories » (consulté le 20/08/19).

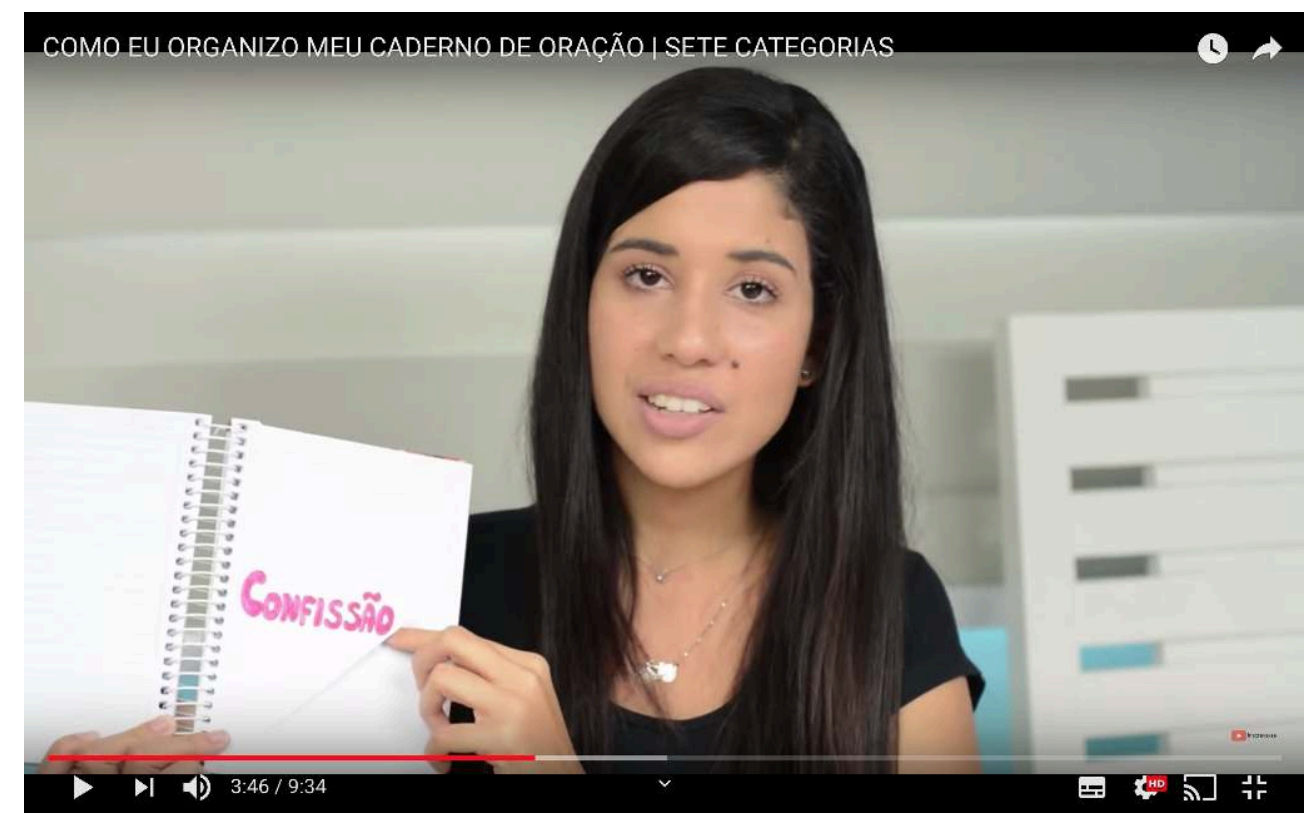

\section{De la sacralisation à la médiatisation d'une ambiance de prière}

Bien que la pratique dévotionnelle s'inscrive dans un rite plus ou moins régulier - lire les Écritures, méditer sur la parole de Dieu, transcrire et écrire des prières l'ambiance de la prière s'instaure de manière plurivoque. Cette ambiance «ne descend pas toujours du ciel comme un phénomène absolument autonome: elle dépend des individus qui la composent». (Bégout, 2018 : 85). Cette ambiance est donc façonnée dans l'espace physique de la prière : le plus souvent, dans la chambre des youtubeuses, dans laquelle se réalise la pratique dévotionnelle. L'on ne prie pas dans un espace neutre et blanc ${ }^{12}$. Le recueillement et la communication avec Dieu ne peuvent se faire que lorsque le croyant rentre en soi, lorsqu'une " ambiance » favorable est instaurée à cet effet. Cette ambiance n'est pas seulement l'endroit où les vidéos s'enregistrent, mais correspond en quelque sorte à un sanctuaire qui résulte d'un «déploiement hétérotopique de l'espace (Salatko, 2017 : 144) ». En effet, c'est l'agencement de l'espace dans cette quête d'esthétisation d'une ambiance de prière qui favoriserait la « communication » avec le dieu chrétien et qui la rend, par conséquent, « sacrée ». Afin de favoriser cette communication, il importe donc d'établir ce que nous pouvons appeler une "ambiance de prière " ou une ambiance sacrée. Faut-il encore préciser, tout d'abord, ce que nous entendons par «sacré » et par «ambiance » et essayer de saisir quels sont éléments susceptibles de «sacraliser » cette ambiance. Le sacrée est compris est compris ici comme ce qui appartient à un domaine de l'intangible et inviolable, dont on confère souvent un caractère solennel, en opposition au profane : "Les choses sacrées sont celles que les interdits protègent et isolent ; les choses profanes, celles auxquelles ces interdits s'appliquent et qui doivent rester à distance des premières " (Durkheim, $1985: 56$ ). Or, si nous suggérons l'idée d'une ambiance sacrée, nous pouvons considérer, d'emblée, que coexiste au moins une autre ambiance : 
une ambiance qui soit profane, donc opposée à la première. L'ambiance, quant à elle " s'adosse à la sensorialité et à l'expérience vécue ", (Thibaud, $2012: 171)$ à la différence d'un environnement, qui renvoie quant à lui, à une notion « d'équilibre » et regroupe l'ensemble d'éléments biotiques ou abiotiques qui « entourent » quelque chose.

19 Au-delà de la vue (pour lire la «parole de Dieu»), du toucher (feuilleter son cahier ou sa Bible, écrire) ou de l'odorat (sentir l'odeur du papier), les jeunes youtubeuses peuvent avoir recours à l'ouïe afin de favoriser une ambiance de communication avec le dieu chrétien. Pour mieux "écouter» le Christ, - pour faire écho aux termes de Francine Veríssimo - les youtubeuses peuvent avoir recours à une musique de fond durant la pratique dévotionnelle (à condition que la musique soit douce). Ainsi, Michelle Souza explique qu'il est préférable, lors de la réalisation de la pratique dévotionnelle, de "mettre une louange, mettre une musique tranquille, une musique calme" $\left(15^{\prime} 41^{\prime \prime}-15^{\prime} 45^{\prime \prime}\right)$. Elle explique avoir une préférence pour les œuvres des chanteurs de musique chrétienne contemporaine Gabriela Rocha et Leonardo Gonçalves. Tainá Brito, pour sa part, explique préférer des chansons en anglais: "J'aime lire [la Bible] en écoutant des chansons en anglais et j'aime beaucoup les chansons [du groupe] Béthel pour écouter lorsque je fais la dévotion » (02'26"-02:34").

20 En ce qui concerne les horaires de la pratique dévotionnelle, tout comme Michelle, Tainá Brito explique qu'il est préférable d'opter pour le matin : «La première chose [à faire] de la journée, c'est de chercher Dieu avant tout »00'42"-00'46"). La notion que nous proposons d'« ambiance sacrée » renvoie à la notion d'espace figuratif du sacré (Dufour \& Boutaud, 2013: 21) qui, par sa dimension figurative, "offre un plan de l'expression au sacré, à travers des signes, des objets, des espaces, des moments qui prennent, se matérialisent dans des gestes, des rites, des actions et engagent les acteurs à performer » (Dufour \& Boutaud, 2013: 21-22). Cette ambiance sacrée, une fois captée ou enregistrée, est ensuite technologiquement modifiée lors de l'édition des vidéos grâce à l'usage des dispositifs socio-numériques conçus à cet effet. Mais, le "sacrée " n'englobe pas seulement des divinités ou les êtres surnaturelles, mais une multiplicité d'éléments: " un rocher, un arbre, une source, un caillou, une pièce de bois, une maison en un mot une chose quelconque peut être sacrée » (Durkheim, 1885 : 56). Cette ambiance sacrée est donc formée à partir de la présence humaine, à savoir, les youtubeuses, ainsi que par les objets ordinaires du quotidien soigneusement assemblés qui prennent part à la pratique dévotionnelle. Les youtubeuses sont donc responsables de la «performation du sacré " puisqu'elles mettent en scène leur "pouvoir de figuration pour donner force et vigueur au sacré, sous des formes non routinisées mais ritualisées, codifiées » (Dufour \& Boutaud, 2013 : 17). Pour ce qui est des objets ou outils d'appui à la prière, nous retrouvons le cahier de prières même, des crayons, des stylos, des trombones, des livres conçus comme guides pour la pratique dévotionnelle, des bibles de versions variées, parfois commentées, telles que la Bible de l'étudiant ou des bibles produites et conçues exclusivement pour les femmes ${ }^{13}$. Dans une approche communicationnelle, le sacré «trouve à s'incarner, au sens premier de 'prendre corps', se faire chair, dans des objets et des mythes, à se déployer à travers des postures, des jeux et des rites, qui l'inscrivent dans une esthétique figurative » (Dufour \& Boutaud, $2013: 21)$.

21 La question qui se pose ensuite est de savoir dans quelle mesure ces captations vidéo peuvent «transmettre " à l'internaute cette «ambiance » à travers la plateforme YouTube. En ce sens, nous pouvons considérer que la technique est en elle-même 
« évangélisée » (Douyère, 2011) et mise au service d'une pédagogie de la prière à travers l'usage des cahiers de prières et de la pratique dévotionnelle quotidienne. En outre, le choix et l'insertion (ou l'absence) d'une musique, le découpage de la vidéo ou l'insertion d'une vignette et l'identité visuelle de la chaine YouTube contribuent à " donner » une ambiance sacrée à cette pratique dévotionnelle médiatisée sur la plateforme internet YouTube. Cependant, n'ayant pas assisté au processus d'édition des vidéos, il nous est impossible de comprendre davantage les choix motivés par des questions religieuses des youtubeuses lors de cette étape créative. De véritables questions qui resteront sans réponse peuvent se poser à propos de ce processus : y a-t-il eu des parties coupées ? Si oui, lesquelles et pourquoi ? Comment s'est fait le choix d'une musique de fond plutôt qu'une autre? Quelles sont les applications ou dispositifs numériques utilisés dans ce processus d'édition, et pourquoi? Auraient-elles prié le Saint-Esprit pour qu'il puisse leur apporter du "discernement", afin qu'elles puissent éditer les vidéos selon « l'image de la volonté de Dieu »? - pour faire écho aux mots de Michelle Souza. En ce sens, l'internet a promu une évolution dans la manière de pratiquer la foi et de voir Dieu : «Dieu ou le sacré doit être engendré, re-signifié, relu, présenté à nouveau, transformé en un processus informatique-ordinateur-communication " (Sbardelotto, 2012 : 133). Cette «liturgie numérique " transforme donc les pratiques religieuses et relève des questions liées au temps et à l'espace.

Les dynamiques ayant pour but de favoriser la ( $\mathrm{re}$ ) diffusion massive par les internautes afin de faire circuler des contenus info-communicationnels, tels que la quête de "like " et de demandes de partage de contenu (pratique récurrente notamment des réseaux sociaux numériques) sont présentes dans les vidéos produites et diffusées par les youtubeuses étudiées. À ce propos, Tainá Brito fait appel aux internautes : «Si vous avez aimé cette vidéo, n'oubliez pas de laisser un «j'aime», ok ? Mettez beaucoup de 'j'aime', s'il vous plait, ok ? Parce que ça sera une bénédiction dans ma vie, dans votre vie, dans notre vie et dans la vie de tout le monde " (07'23"-07' 35"). Elle demande encore aux internautes de s'abonner à sa chaine YouTube et de partager la vidéo dans leurs réseaux sociaux. Fabíola Keffer demande à l'internaute de laisser des commentaires à propos de sa vidéo : « dites ci-dessous si vous avez fait cette dévotion avec moi. Dites ci-dessous ce que vous avez appris de ce texte, de cette dévotion d'aujourd'hui. Amen?» (19'11"-19'27"). De la même manière, Sarah Néry sollicite l'interaction des internautes: "mettez un 'j'aime' si vous avez aimé cette vidéo et n'oubliez pas de vous inscrire à la chaîne [...] et invite un autre ami à s'inscrire aussi pour que nous puissions grandir et que plus de monde puisse être touché par la miséricorde de Dieu (09'14"-09' : 30"), dit-elle.

Sur le plan de l'interaction avec les internautes, les youtubeuses Fabíola Keffer et Michelle Souza invitent l'autre à prier avec elle : «avant tout, je veux t'inviter à prier avec moi » (00' 32"-01'20"). Et « on va faire donc cette petite prière à Dieu maintenant, amen ? (08'21"-09 '10"). Tout au long de la prière, l'image est portée en noir et blanc, ce qui donne à l'ambiance une tonalité plus réservée et sobre. Enfin, des phénomènes de (re)négociations entre pratiques religieuses traditionnelles qui ont lieu habituellement hors de l'espace numérique peuvent s'ajuster à l'environnement numérique (Campbell, 2010). En ce sens, la présence d'un ascétisme chrétien en contexte numérique (Jonveaux, 2007) peut aussi être observée. Tainá Brito explique avoir incorporé comme devise dans sa vie quotidienne, le hashtag "\#SemDovicionalSemRedeSocial» (sans dévotion, pas de réseaux sociaux [numériques]). Ainsi, nous pouvons constater que dans la conception de la youtubeuse, la pratique dévotionnelle quotidienne conditionne 
l'usage des réseaux sociaux numériques qui sont compris ainsi comme des dispositifs qui renvoient à une notion de plaisir compte tenu de leur caractère ludique. En ce sens, les expériences et vécus de la religion sur internet ne consistent pas simplement à cliquer sur des hyperliens pour consommer des contenus informationnels religieux (Helland, 2005) mais configurent des dynamiques comme des formes esthétiques et relèvent de subjectivations religieuses complexes.

\section{Conclusion}

Nous avons mené une analyse concernant des vidéos, tutoriels de prière instrumentée, manifestant un processus de mise en scène d'une ambiance sacrée. Ces productions audiovisuelles ont clairement pour ambition d'inciter les internautes à élaborer un cahier de prières dans le cadre de leur pratique dévotionnelle quotidienne. Cette pratique prend place dans une ambiance à portée polysémique: elle "prend» son caractère "sacré ", d'une part, de la mise en scène de la présence humaine incarnée, à savoir les youtubeuses, mais aussi des objets ordinaires variés (crayons, bibles, les cahiers de prière même) et, d'autre part, lors du processus d'édition de la vidéo à l'appui des dispositifs numériques créés à cet effet (choix de la musique, position de la caméra, etc.). Tout d'abord, une ambiance ne peut sans doute être instaurée par la seule intervention humaine. Ici, c'est la présence et la performation du sacré exercée par les acteurs qui rendent cette ambiance sacrée. Ensuite, certains de ces objets ordinaires peuvent être même compris comme des artefacts de piété, puisque ces objets ont une fonction précise : appuyer la pratique dévotionnelle, assister la prière ou «rendre beau » le cahier de prières. Ce sont donc des objets qui non seulement font partie intégrante de l'espace figuratif du sacré ou de l'ambiance sacrée, mais qui participent même à la sacralisation de cette ambiance. Ce qui est précisément le cas, quand bien même ils n'auraient pas été conçus à cette fin, du chapelet ou du scapulaire chrétiens. Bien que le processus d'édition des vidéos ne nous soit pas accessible, il a bien eu lieu et il fait partie intégrante de la médiatisation de cette ambiance sacrée. Les décisions prises dans ce processus relèvent, naturellement, des questions de compétence ou d'aisance liées à la technologie, des goûts dans la quête d'une esthétique. En ce sens, la religion ou le religieux n'échappe pas aux logiques et "codes " caractéristiques de ce que nous appelons les «industries créatives ». Cette logique s'inscrit notamment dans un paradoxe entre la standardisation des dispositifs numériques et une certaine originalité des contenus proposés par les internautes. Cependant, ce processus d'édition et les prises de décisions qui s'y inscrivent peuvent même être pensés comme une véritable expérience religieuse, puisqu'il est motivé par des croyances et des perspectives religieuses. 


\section{BIBLIOGRAPHIE}

BEGout Bruce (2018). «L'ambiance comme aura : Le pouvoir atmosphérique des individualités », Communications, 102 (1), pp. 81-98.

CAMPBELl Heidi \& VITULLo Alessandra (2016). « Assessing changes in the study of religious communities in digital religion studies ", Church, Communication and Culture, 1 (1) [en ligne], mis en ligne 26.05.16, consulté 11.04.19. URL : https://www.tandfonline.com/doi/full/ $10.1080 / 23753234.2016 .1181301$.

CAMPBELL Heidi (2005). Exploring religious community online: We are one in the network, New York, Peter Lang.

CAMPBELL Heidi (2010). When religion meets new media, London, New York, Routledge.

CARDON Domnique (2015). À quoi rêvent les algorithmes. Nos vies à l'heure des big data, Paris, Le Seuil.

CHRISTIN Olivier (2002). « Du culte chrétien au culte de l'art : la transformation du statut de l'image (XVe-XVIIIe siècles) », Revue d'histoire moderne et contemporaine, 49 (3) [en ligne], consulté 50.04.2019. URL : https://www.cairn.info/revue-d-histoire-moderne-et-contemporaine-2002-3page-176.htm.

DE KonincK Ralph (2007). « Ars symbolica et ars meditandi », Littérature, 1, p. 105-118.

DOUYERE David (2011). « La prière assistée par ordinateur », Médium, 27 (2) [en ligne], mis en ligne 01.02.13, consulté 13.05.19. URL : https://www.cairn.info/revue-medium-2011-2-page-140.htm.

DOUYERE David (2014) « Communiquer la prière par l'image du corps : les 'Neuf manières de prier de saint Dominique', une représentation incitative et identitaire de la prière et du recueillement », in Lambert Frédéric (dir.), Prières et propagandes, études sur la prière dans les arènes publiques, Paris, Hermann.

DOUYERE David (2018). Communiquer la doctrine catholique: Textes et conversations durant le concile Vatican II d'après le journal d'Yves Congar, Genève, Labor et Fides.

DUFOUR Stéphane \& BOUTAUD, Jean-Jacques (2013). « Extension du domaine du sacré ». Questions de communication, 23 [en ligne], consulté 01.08.2019. URL : https://www.cairn.info/revue-questionsde-communication-2013-1-page-7.htm

DURKHEIM Émile (1985). Les Formes élémentaires de la vie religieuse, Paris, PUF.

GAUCHET Marcel (1981) « Des deux corps du roi au pouvoir sans corps. Christianisme et politique », Le débat (14) 7, pp.133-157.

Helland Christopher (2005). « Online religion as lived religion. Methodological issues in the study of religious participation on the Internet », Online-Heidelberg Journal of Religions on the Internet, 1 (01) [en ligne], mis en ligne 22.09.05, consulté 17.04.19. URL : http://www.ub.uni-heidelberg.de/ $\operatorname{archiv/5823.~}$

HERVIEU-LEGER Danièle (1997). « La transmission religieuse en modernité: éléments pour la construction d'un objet de recherche », Social Compass 44 (1), pp. 131-143.

HERVIEU-LEGER, Danièle (2010). « Le partage du croire religieux dans des sociétés d'individus », L'Année sociologique 60 (1), pp. 41-62. 
JONVEAUX Isabelle. (2007). « Une retraite de carême sur Internet », Archives de sciences sociales des religions (139), 157-176.

MAUSS Marcel (1968). La Prière, Paris, Éditions de Minuit.

ORIGENE (2012). Tatato sobre os principios, São Paulo, Paulus.

PALAZzo Eric (2016). Peindre, c'est prier : anthologie de la prière chrétienne, Paris, Editions du Cerf.

PALAZzo Eric (2017) «La liturgie dévotionnelle et les cinq sens : les neuf modes de prière de saint Dominique », Perspectives médiévales, 38 [en ligne], mis en ligne 01.01.17, consulté 26.04.19. URL : https://journals.openedition.org/peme/12269\#quotation.

PIERRON Jean-Philippe (1995). « La question du témoignage dans les Confessions de saint Augustin », Revue d'études augustiniennes et patristiques, 41 (2), pp. 253-266.

SALATKO Gaspard (2017). Le dieu situé: une enquête sur la fabrique de l'art sacré dans le catholicisme contemporain, Paris, L'Harmattan.

SANTANA Josineide Siqueira de (2011). « Entre bordados, cadernos e orações: a educação de meninas e as práticas educativas no orfanato de São Cristóvão e na escola da Imaculada Conceição (1922-1969) », Mémoire de master en Sciences de l'éducation sous la direction de FREITAS, Anamaria Gonçalves Bueno de, Universidade Federal de Sergipe [en ligne], mis en ligne 01.04.11, consulté 01.04.19. URL : http://bdtd.ibict.br/vufind/Record/

UFS-2_71c95f8a5e72cbbe291a48704326fb63.

SEVEz Pascal (2018). Pédagogie ignacienne. Études, (1) pp. 59-80, [en ligne], mis en ligne 03.01.2018, consulté 16.08.19. URL : https://www.cairn.info/revue-etudes-2018-1-page-69.htm

STEIL Carlos Alberto \& TONIOL Rodrigo (2013). « A crise do conceito de religião e sua incidência sobre a antropologia ». Giumbelli Emerson \& Béliveau Verónica Gimenéz. (dir.) Religión, cultura y política en las sociedades del siglo XXI, Buenos Aires, Biblos editora, pp. 137-158.

THIBAUD Jean-Paul (2018). « Les puissances d'imprégnation de l'ambiance ». Communications, 102 (1), pp. 67-79.

THIBAUD Jean-Paul (2012). « Petite archéologie de la notion d'ambiance », Communications, 90, pp. 155-174.

TROTTMANN Christian (2003). « Contemplation et vie contemplative selon trois Chartreux : Guigues II, Hugues de Balma et Guigues du Pont: Quelques points de repère dans une évolution ». Revue des sciences philosophiques et théologiques, 87(4), pp. 633-680.

VANOYE Francis \& GOLIOT-LETE Anne (2006). Ensaio sobre a análise filmica, Campinas, Papirus.

\section{NOTES}

1. Nous avons choisi de garder la traduction littérale du portugais brésilien cadernos de oração, bien qu'en français, il serait plus précis de parler de journal intime de prière afin de le distinguer des cahiers de prières publics que nous trouvons, le plus souvent, dans les églises. Nous remercions vivement Andrea Castellani de nous en avoir fait la remarque. Nous tenons aussi à préciser que les traductions du portugais brésilien vers le français ont été réalisées par nousmême.

2. Il s'agit d'une communauté charismatique catholique créée au Brésil dans les années 1980. 
3. Au Brésil, face aux nombreuses filiations dites "protestantisme traditionnel » et la multiplication de filiations chrétiennes - notamment pentecôtistes et néo-pentecôtistes certains auteurs qui étudient les religions ou le «religieux» dans le pays considèrent «évangéliques" (évangélicos) tout fidèle chrétien qui n'est pas catholique romain ni chrétien orthodoxe. En effet, de très nombreuses affiliations chrétiennes diverses et variées voient le jour tous les jours dans des hangars ou garages dans le pays. Ainsi, il est courant de " penser » les « évangéliques » de manière large, quand bien même il s'agit de groupes très hétérogènes.

4. Repéré à https://www.youtube.com/watch?v=wZm0oGpFqVE. Consulté le 25.05.2019.

5. Repéré à https://www.youtube.com/watch?v=90IpxPq7ubU\&t=2s. Consulté 04.04.2019.

6. Repéré à https://www.youtube.com/watch?v=BMoul58kU0Q. Consulté le 02.04.2019.

7. Repéré à https://www.youtube.com/watch?v=TxXUtlI7DFE\&t=198s. Consulté 02.04.2019.

8. Il s'agit de l'abréviation du mot acompanhantes, qui pourrait être traduit par accompagnateur en français.

9. Repéré à https://www.youtube.com/watch?v=Gz5pVaQccM0. Consulté 12.04.2019.

10. Repéré à https://www.youtube.com/watch?v=UqkSiwjyTWI\&t=1s. Consulté 05.04.2019.

11. Repéré à https://www.youtube.com/watch?v=C88PomifydE\&t=156s. Consulté 10.04.19.

12. Nous détournons ici la phrase "on ne vit pas dans un espace neutre et blanc » de Michel Foucault lors du cours Les Hétérotopies, sur France-Culture, le 7 décembre 1966.

13. Des bibles telles que "la Bible de l'enfant", «la bible de l'adolescent », « la bible pour le couple » et des versions diverses et variées de la bible destinées à des différents publics sont proposées par une très vaste et florissante industrie religieuse chrétienne depuis ces dernières années au Brésil.

\section{RÉSUMÉS}

Cette recherche s'intéresse au processus de mise en scène d'une « ambiance sacrée " à travers des productions audiovisuelles publiées et diffusées par de jeunes femmes laïques chrétiennes brésiliennes sur la plateforme YouTube. À partir des productions qui ont pour ambition d'apprendre aux internautes à créer un cahier de prières, une ambiance sacrée s'élabore en deux temps : d'une part, à partir de l'assemblage d'objets ordinaires du quotidien et dans un espace physique qui lui est réservé, correspondant le plus souvent à la chambre des youtubeuses ; d'autre part, lors de l'édition de vidéos grâce à l'usage des dispositifs socio-numériques conçus à cet effet. Bien que s'inscrivant dans une démarche pédagogique d'incitation à une pratique de piété populaire, ces productions audiovisuelles cherchent également à donner à voir un «éthos " de l'idéal de la femme chrétienne ainsi que de parler du dieu chrétien dont elles sont les interlocutrices.

This research focuses on the process of staging a « sacred ambiance » from videos published and broadcast by Brazilian Christian Laywomen on the Youtube platform. From the productions that aim to teach Internet users to elaborate a prayer book, a sacred ambiance is developed in two stages: on one hand, from the assembly of ordinary everyday objects and into a proper physical space that most often corresponds to the bedroom of Youtubers; on the other hand, when editing videos through the use of socio-digital devices designed for this purpose. Although they are part of a pedagogical approach of incitement to a practice of popular piety, these audiovisual 
productions also seek to show an « ethos » of the ideal Christian woman as well as to speak of the Christian god of which they are the interlocutors.

INDEX

Mots-clés : Cahier de prières, Youtube, vidéo, sacré, prière, dévotion, religion.

Keywords : Youtube, video, sacred, prayer, devotion, religion

\section{AUTEUR}

\section{KELBER PEREIRA GONÇALVES}

Université de Tours, Pim 